Philosophical Magazine A, 1986, Vol. 53, No. 6, 783-791

\title{
Lowest-order approximations to relaxation volumes of monovacancies in cubic metals from pair potentials and Finnis-Sinclair potentials
}

\author{
By W. MAYSENHÖLDER \\ Universität Stuttgart, Institut für Theoretische und Angewandte Physik, Pfaffenwaldring 57, \\ D-7000 Stuttgart 80, F.R. Germany, and Max-Planck-Institut für Metallforschung, \\ Institut für Physik, Heisenbergstr., I, D-7000 Stuttgart 80, F.R. Germany
}

[Received 5 November 1985 and accepted 20 November 1985]

ABSTRACT

Relaxation volumes of point defects in cubic metals can be conveniently estimated by differentiating approximate formation energies with respect to the lattice constant. Lowest-order analytical expressions for monovacancies have been derived for equilibrium pair potentials and for the $\mathrm{N}$-body potentials of Finnis and Sinclair (1984). These expressions may be evaluated with little effort and prove far superior to other lowest-order approximations to vacancy relaxation volumes.

\section{$\S 1$. INTRODUCTION}

Vacancy relaxation volumes provide a stringent test of the quality of interatomic potentials which are supposed to yield reliable information about lattice defects. A classical example is the monovacancy in copper, for which all central equilibrium pair potentials failed to reproduce the experimental relaxation of about -0.2 atomic volumes. It was necessary to include many-body interactions in order to solve this problem (cf. Bauer, Maysenhölder and Seeger 1982). Accurate relaxation is also very important for calculations of vacancy formation entropies to be reliable, because the vibrational frequencies depend critically on the atomic positions (cf. Hatcher, Zeller and Dederichs 1979, Maysenhölder, Bauer and Seeger 1985).

Nevertheless, some authors avoid the computational effort needed for relaxation volumes and calculate only relaxation energies and displacements of near neighbours to the vacancy. To demonstrate that this effort can be kept quite small we present in $\S 2$ a concise formulation for determining relaxation volumes of point defects in cubic metals from interatomic potentials, which is readily implemented in computer simulations using rigid boundary conditions. Starting from this formulation, which essentially goes back to Finnis and Sachdev (1975) and Schober and Ingle (1980), we derive analytical lowest-order approximations to monovacancy relaxation volumes in a systematic way. Since relaxation volumes of vacancies are usually small and much less than one atomic volume, we expect these estimates to come close to exact resuits.

The general expressions of $\$ 2$ are evaluated for equilibrium pair potentials in $\S 3$ and for the potentials of Finnis and Sinclair (1984) in $\S 4$. In the final section ( $\$ 5$ ) comparison is made with other lowest-order approximations, which turn out to provide mostly poor results. 


\section{§2. METHOD}

We consider a point defect in an infinite cubic lattice, which we divide into two regions: region I containing the first $N$ shells of atoms around the point defect, and region II containing all other atoms, which are supposed to be fixed to the unrelaxed positions of the corresponding perfect lattice. The atoms in region I are allowed to relax, their equilibrium positions being dependent on the positions of the atoms in region II, i.e. dependent on the lattice constant. The energy of this configuration minus the energy of the corresponding perfect crystal with the same number of atoms is called the $N$ th approximation $E^{(N)}$ to the point-defect formation energy

$$
E^{\bar{F}}=\lim _{N \rightarrow \infty} E^{(N)} \equiv E^{(\infty)}
$$

$E^{(0)} \equiv E_{0}^{\mathrm{F}}$ denotes the formation energy in a rigid lattice.

The relaxation volume $V_{\text {rel }}$ of a point defect in a cubic crystal is obtained from the elastic dipole tensor $\mathbf{P}$ and the bulk modulus $B$ according to

$$
V_{\text {rel }}=\operatorname{tr} \mathbf{P} /(3 B)
$$

(Leibfried and Breuer 1978, p. 149). It will be shown that

$$
\operatorname{tr} \mathbf{P}=-a \frac{\mathrm{d} E^{(\infty)}}{\mathrm{d} a}
$$

We observe that the force $F^{(l)}$ on atom $l$ at position $x^{l}$,

$$
F_{j}^{(l)}=-\frac{\partial E^{(\infty)}}{\partial x_{j}^{(l)}},
$$

is non-zero only for atoms $l=m$ lying in a mantle around region $I$ with a thickness equal to the range of the interatomic interaction. In the limit $N \rightarrow \infty$ these forces become equal to the Kanzaki forces (Kanzaki 1957); therefore, according to the standard expression

$$
\begin{gathered}
P_{i j}=\sum_{m} x_{i}^{(m)} F_{j}^{(m)}, \\
\operatorname{tr} \mathbf{P}=-\sum_{m, i} x_{i}^{(m)} \frac{\partial E^{(\infty)}}{\partial x_{i}^{(m)}}=-\sum_{m} r^{(m)} \frac{\partial E^{(\infty)}}{\partial r^{(m)}}
\end{gathered}
$$

with $r^{(m)}=\left|x^{(m)}\right|$. Because all atoms $m$ are in unrelaxed positions, $r^{(m)}$ is some multiple of the lattice constant $a$, and the identity

$$
a \frac{\mathrm{d} E^{(\infty)}}{\mathrm{d} a}=a \sum_{m} \frac{\partial E^{(\infty)}}{\partial r^{(m)}} \frac{\mathrm{d} r^{(m)}}{\mathrm{d} a}=\sum_{m} r^{(m)} \frac{\partial E^{(\infty)}}{\partial r^{(m)}}
$$

completes the proof of eqn. (2). Finally, the formation volume of the defect reads

$$
V^{\mathrm{F}}=n \Omega+V_{\mathrm{rel}}=n \Omega-\frac{a}{3 B} \frac{\mathrm{d} E^{(\infty)}}{\mathrm{d} a}
$$

(A multiple of the atomic volume $\Omega$ has been added to $V_{\text {rel }}$; for a vacancy $n=1$, for an interstitial $n=-1$.) 
We note in passing that the thermodynamic relation $V^{\mathrm{F}}=\partial H^{\mathrm{F}} / \partial P$ between the formation volume $V^{F}$ and the pressure derivative of the formation enthalpy $H^{\mathrm{F}}$ can be transformed into

$$
V^{\mathrm{F}}=\frac{\partial H^{\mathrm{F}}}{\partial \Omega} \frac{\partial \Omega}{\partial P}=-\frac{a}{3 B} \frac{\partial H^{\mathrm{F}}}{\partial a} .
$$

(Partial derivatives imply constant zero temperature.) Thus, from a thermodynamic point of view, $E^{(\infty)}+n P \Omega$ has to be considered a formation enthalpy rather than an internal energy. This is because the interaction between the 'system' (region I) and the 'pressure bath' (region II) is contained in $E^{(\infty)}$. (The 'constant volume formation energy' defined by Finnis and Sachdev (1976) is equivalent to $E^{(\infty)}+n P \Omega$ and should also be called an enthalpy.)

Equation (7) with $\infty$ replaced by some finite number $N$ can readily be employed in computer simulations of point defects in cubic lattices (particularly if rigid boundary conditions are used). We expect rapid convergence with increasing $N$.

For a vacancy, the lowest-order approximations $V_{\mathrm{rel}}^{(N)}$ already constitute reasonable estimates of the relaxation volume. $N=0$ means vanishing region I, no atoms relaxed, i.e.

$$
V_{\mathrm{rel}}^{(0)}=-\frac{a}{3 B} \frac{\mathrm{d} E_{1 V, 0}^{\mathrm{F}}}{\mathrm{d} a}
$$

For $N=1$ the nearest neighbours to the vacancy relax and suffer relative displacements $\delta$. If we restrict our analytical treatment to harmonic terms, $\delta$ minimizes the expression

$$
\delta E_{\delta}+\frac{1}{2} \delta^{2} E_{\delta \delta}
$$

(superscript 1 suppressed; subscript $\delta$ denotes differentiation at $\delta=0$ with $a$ fixed), i.e.

$$
\delta=-\frac{E_{\mathrm{\delta}}}{E_{\delta \delta}}
$$

Therefore

$$
E^{(1)}=E_{1 V, 0}^{\mathrm{F}}-\frac{E_{\delta}^{2}}{2 E_{\delta \delta}}
$$

and

$$
V_{\mathrm{rel}}^{(1)}=V_{\mathrm{rel}}^{(0)}+\frac{a}{3 B} \frac{E_{\delta}}{E_{\delta \delta}} \frac{\mathrm{d} E_{\delta}}{\mathrm{d} a}
$$

For $N=2$ we obtain

$$
V_{\mathrm{rel}}^{(2)}=V_{\mathrm{rel}}^{(0)}+\frac{a}{3 B}\left(\begin{array}{l}
E_{\delta} \\
E_{\varepsilon}
\end{array}\right)\left(\begin{array}{ll}
E_{\delta \delta} & E_{\delta \varepsilon} \\
E_{\delta \varepsilon} & E_{z \varepsilon}
\end{array}\right)^{-1} \frac{\mathrm{d}}{\mathrm{d} a}\left(\begin{array}{c}
E_{\delta} \\
E_{\varepsilon}
\end{array}\right)
$$

with $\varepsilon$ denoting the relative displacement of next-nearest neighbours. The generalization of eqn. (13) to $N>2$ is straightforward but not very useful since anharmonic effects are presumably more important than relaxation of more distant shells of atoms. In fact, we restrict ourselves to $V_{\mathrm{rel}}^{(0)}$ and $V_{\mathrm{rel}}^{(1)}$ for the evaluation of specific potentials in the following sections. 


\section{§3. APPLICATION TO EQUILIBRIUM PAIR POTENTIALS}

The equations of $\$ 2$ hold for general interatomic potentials in cubic lattices. Now we assume a pairwise interaction potential $V(r)$ for which the volume derivative of the cohesive energy per atom $E_{\text {coh }}$ vanishes at zero pressure (equilibrium pair potential). Since $E_{1 v, 0}^{\mathrm{F}}=-E_{\mathrm{coh}}$, we arrive at

$$
V_{\mathrm{rel}}^{(0)}=0
$$

(for zero pressure). If the range of $V(r)$ is restricted to first and second neighbours, the expressions needed for $V_{\mathrm{rel}}^{(1)}$ read for a vacancy in a b.c.c. lattice:

$$
\begin{aligned}
E_{\mathrm{d}} & =-8 W_{1}, \\
E_{\mathrm{dd}} & =\frac{2}{3}\left(64 W_{1}+18 W_{2}+20 \tilde{W}_{1}+27 W_{2}\right), \\
a \frac{\mathrm{d} E_{\mathrm{d}}}{\mathrm{d} a} & =-8\left(W_{1}+W_{1}\right)
\end{aligned}
$$

with $(i=1,2)$

and

$$
W_{i}=\left.r_{i} \frac{\mathrm{d} V}{\mathrm{~d} r}\right|_{r_{i}}, \quad \tilde{W}_{i}=\left.r_{i}^{2} \frac{\mathrm{d}^{2} V}{\mathrm{~d} r^{2}}\right|_{r_{i}}
$$

$$
r_{1}=\frac{1}{2} \sqrt{ } 3 a, \quad r_{2}=a
$$

For the f.c.c. case we have

$$
\begin{aligned}
E_{\delta} & =-12 W_{1}, \\
E_{\mathrm{d} \delta} & =6\left(10 W_{1}+3 W_{2}+8 W_{1}+3 W_{2}\right), \\
a \frac{\mathrm{d} E_{\mathrm{\delta}}}{\mathrm{d} a} & =-12\left(W_{1}+W_{1}\right)
\end{aligned}
$$

with the same meaning of $W_{i}$ and $W_{i}$ as above except for the different nearest-neighbour distance $r_{1}=\frac{1}{2} \sqrt{ } 2 a$.

Two examples may illustrate the relatively small vacancy relaxation produced by equilibrium pair potentials. For the $\alpha$-iron potential of Johnson (1964) we get $\delta=-1.9 \%, \quad E_{\mathrm{rel}}^{(1)}=E^{(1)}-E_{1 V .0}^{\mathrm{F}}=-5.6 \mathrm{meV}$, and (with $B=0.800 \mathrm{eV} \AA^{-3}$ ) $V_{\mathrm{rel}}^{(1)}=$ $-0.08 \Omega$. (Unfortunately, Do value for $V_{\text {ret }}$ of the fully relaxed configuration could be found in the literature.) Evaluation of the modified Morse potential introduced by Schober and Zeller (1978) yields $\delta=-0.3 \%, E_{\mathrm{rel}}^{(1)}=-4 \mathrm{meV}$, and $V_{\mathrm{rel}}^{(1)}=-0.02 \Omega$. The last number is in full agreement with the value for $V_{\text {rel }}$ given by Dederichs, Lehman, Schober, Scholz and Zeller (1978).

\section{§4. Application to Finnis-Sinclatr potentials}

The potentials of Finnis and Sinclair (1984) for the b.c.c. transition metals have opened new perspectives for computer simulations of lattice defects. The most attractive feature is the inclusion of many-body interactions without any significant increase of computational effort over usual pair-potential calculations. The secondorder elastic constants can be fitted exactly even for non-zero Cauchy pressure $P_{\mathrm{c}}=\frac{1}{2}\left(C_{12}-C_{44}\right)$. Further, $E_{1 V, 0}^{\mathrm{F}} \approx E_{1 V}^{\mathrm{F}}$ is no longer equal to $-E_{\mathrm{coh}}$ but considerably less, as experimentally observed. If desired, both $E_{\text {cob }}$ and $E_{1 v}^{\mathrm{F}}$ can be fitted to experimental data with minor modifications of the fitting procedure. 
It remains to be seen how well experimental vacancy relaxation volumes (when available) can be reproduced. Matthai and Bacon (1985) have calculated relaxation energies, but no relaxation volumes; only the relative displacements of first and second neighbours are given. For estimates of vacancy relaxation volumes in these metals we evaluate the lowest-order approximations of $\S 2$ for the Finnis-Sinclair potentials. (Unless otherwise stated, the notation is the same as in the original paper of Finnis and Sinclair (1984), which is referred to as FS in the following.)

The vacancy formation energy in a rigid b.c.c. lattice given by FS,

$$
E_{1 V, 0}^{\mathrm{F}}=-A\left(8 f_{1}+6 f_{2}-14 f\right)-u_{P}
$$

with

$$
\begin{gathered}
f=\left(8 \Phi_{1}+6 \Phi_{2}\right)^{1 / 2} \\
f_{1}=\left(7 \Phi_{1}+6 \Phi_{2}\right)^{1 / 2}, \quad f_{2}=\left(8 \Phi_{1}+5 \Phi_{2}\right)^{1 / 2}
\end{gathered}
$$

can be approximated quite well by

$$
E_{1 v, 0}^{\mathrm{F}} \approx \frac{1}{2} A f-u_{P}=-\left(\frac{1}{2} u_{N}+u_{\mathrm{P}}\right)
$$

provided $v=\left|\Phi_{2} / \Phi_{1}\right| \geqq 1$. For a typical value $v=\frac{1}{2}$ the exact result is obtained by replacing the factor $\frac{1}{2}$ in eqn. (18) by $0.5098 \ldots$... (It should be remembered that the cohesive energy per atom is the sum of the pair-potential term $u_{P}=4 V_{1}+3 V_{2}$ and the $N$-body term $u_{N}=-A\left(8 \Phi_{1}+6 \Phi_{2}\right)^{1 / 2}$, where $A$ is a positive constant; $P_{P}=-\mathrm{d} u_{P} / \mathrm{d} \Omega$ and $P_{N}=-\mathrm{d} u_{N} / \mathrm{d} \Omega$ add up to the external pressure.) Similarly, the lattice-parameter derivative can be calculated from eqn. (18) to within about $2 \%$. This leads to

$$
\frac{V_{\mathrm{rel}}^{(0)}}{\Omega} \approx \frac{1}{2} \frac{P_{N}}{B}
$$

Since at zero pressure $P_{N}+P_{P}=0, V_{\mathrm{rel}}^{\mathrm{r}}$ is generally non-zero in contrast to the pairpotential case of $\S 3$. (Foreman (1963) was probably the first to realize this important effect of many-body interactions.) For the first-order correction $V_{\mathrm{rel}}^{(1)}-V_{\mathrm{rel}}^{(0)}$ we arrive at expressions analogous to eqn. (15), but with some additional terms:

$$
\begin{aligned}
E_{\delta} & =-8 X_{1}, \\
E_{\delta \delta} & =\frac{2}{3}\left(64 X_{1}+18 X_{2}+20 X_{1}+27 X_{2}\right)+2 A f^{-1}\left(4 \Gamma_{1}^{2}-3 \Gamma_{1} \Gamma_{2}+3 \Gamma_{2}^{2}\right), \\
a \frac{\mathrm{d} E_{\delta}}{\mathrm{d} a} & =-8\left(X_{1}+X_{1}\right)-8 A f^{-1}\left(4 \Gamma_{1}+3 \Gamma_{2}\right) \Gamma_{1},
\end{aligned}
$$

with $(i=1,2)$

$$
\Gamma_{l}=r_{i} \Phi_{i}^{\prime} f^{-1}, \quad \Gamma_{i}=r_{i}^{2} \Phi_{i}^{\prime \prime} f^{-1}
$$

and

$$
X_{i}=W_{i}-A \tilde{\Gamma}_{i}, \quad \tilde{X}_{i}=\tilde{W}_{i}-A \tilde{\Gamma}_{i} .
$$

Table 1 shows the results for the group Vb and VIb metals. Finnis and Sinclair (1984) also provide a potential for $\alpha$-iron, but unfortunately it does not match the correct elastic constants; therefore results on Fe could not be included. (The elastic constants of $\alpha$-iron, which are inadvertently given in units of $\mathrm{eV} / \AA^{3}$ in table 1 of FS (C. Teodosiu 1985, personal communication), have erroneously been taken by FS to be in units of $10^{11} \mathrm{~Pa}$.) With the exception of chromium $\left(P_{c} \approx 0\right)$ there is an appreciable 
Table 1. Lowest-order approximations $V_{\text {rel }}^{N N}$ to vacancy relaxation volumes and first-order approximations to relaxation energies and relative displacements of nearest neighbours. In the last two columns corresponding values for the fully relaxed vacancy from Matthai and Bacon (1985) are given.

\begin{tabular}{cccccccc}
\hline Metal & $\begin{array}{c}V_{\mathrm{re}}^{(0)} \\
(\Omega)\end{array}$ & $\begin{array}{c}V_{\mathrm{rel}}^{(1)}(\Omega) V_{\mathrm{rel}}^{(0)} \\
(\Omega)\end{array}$ & $\begin{array}{c}V_{\mathrm{rel}}^{(1)} \\
(\Omega)\end{array}$ & $\begin{array}{c}E_{\mathrm{rel}}^{(1)} \\
(\mathrm{eV})\end{array}$ & $\begin{array}{c}\delta \\
(\%)\end{array}$ & $\begin{array}{c}E_{\mathrm{rel}}^{\mathrm{MB}} \\
(\mathrm{eV})\end{array}$ & $\begin{array}{c}\delta^{\mathrm{MB}} \\
(\%)\end{array}$ \\
\hline $\mathrm{V}$ & -0.25 & -0.06 & -0.31 & -0.04 & -1.8 & -0.14 & -2.5 \\
$\mathrm{Nb}$ & -0.28 & -0.05 & -0.34 & -0.05 & -1.7 & -0.16 & -2.6 \\
$\mathrm{Ta}$ & -0.21 & -0.07 & -0.28 & -0.08 & -1.8 & -0.27 & -3.0 \\
$\mathrm{Cr}$ & -0.03 & -0.02 & -0.05 & -0.02 & -0.7 & -0.06 & -1.1 \\
$\mathrm{Mo}$ & -0.13 & -0.01 & -0.14 & -0.01 & -0.5 & -0.04 & -0.8 \\
$\mathrm{~W}$ & -0.11 & -0.02 & -0.13 & -0.03 & -0.7 & -0.09 & -1.2 \\
\hline
\end{tabular}

zeroth-order contribution to the vacancy relaxation volume. $V_{\text {rel }}^{(1)}$ can be regarded as a reasonable estimate of $V_{\text {rel }}$, if the error is expected not to exceed the first-order correction $\left|V_{\mathrm{rel}}^{(1)}-V_{\mathrm{ref}}^{(0)}\right|$. This expectation is supported by previous calculations for the noble metals (Bauer et al. 1982), where $V_{\text {rel }}^{\mathrm{rol}} / \Omega=-0.15,-0.22$ or -0.25 for $\mathrm{Cu}, \mathrm{Ag}$ or Au compared to the values $-0.21,-0.31$ or -0.37 for the fully relaxed vacancy. In the last four columns of table 1 relaxation energies and relative displacements of nearest neighbours obtained from the present first-order approximation can be compared to the values of Matthai and Bacon (1985) for a fully relaxed vacancy. Apparently, the first-order approximation yields only $\frac{1}{4}$ or $\frac{1}{3}$ of the total relaxation energy and about $\frac{2}{3}$ of the relative displacements of the nearest neighbours. However, we expect $V_{\mathrm{rel}}^{(1)}$ to be closer to $V_{\text {rel }}$, because (i) it relies on the calculation of forces, which are-unlike the relaxation energy - not so strongly dependent on displacements and because (ii) there will be some cancellation of the higher-order corrections in contrast to the relaxation energy, which decreases monotonically with the number of relaxing shells of atoms.

One might argue that the second-order approximation $V_{\mathrm{rel}}^{(2)}$ would be preferable to $V_{\mathrm{rel}}^{\mathrm{rl}}$ for b.c.c. lattices, because first and second neighbours possess very similar distances to the vacancy, but evaluation of eqn. (13) will be quite cumbersome and beyond 'quick estimates', which are the main object of this paper.

No experimental values of $V_{\text {rel }}$ for the metals of table 1 are available yet. There is only an assumption for molybdenum $\left(V_{\text {rel }}=-0.1 \Omega\right)$ made by Ehrhart (1978), which matches the corresponding $V_{\text {rel }}^{(1)}$ in table 1 quite well.

\section{§5. COMPARISON WITH OTHER LOWEST-ORDER APPROXIMATIONS}

The treatment of $\S 3$ can easily be extended to non-equilibrium pair potentials fitted to the elastic constants such that

$$
\frac{\mathrm{d} E_{\mathrm{cob}}}{\mathrm{d} \Omega}=-P_{\mathrm{e}}
$$

Since $E_{1 v, 0}^{F}=-E_{\text {coh }}$ for any pair potential, insertion of eqn. (21) in eqn. (9) gives

$$
\frac{V_{\mathrm{rel}}^{(0)}}{\Omega}=-\frac{P_{\mathrm{c}}}{B}
$$

This result, however, is not in accord with the philosophy of non-equilibrium pair potentials. We rather should decompose $E_{1 V, 0}^{F}=-2 E_{\mathrm{cob}}+E_{\mathrm{cob}}$ (removal of one atom 
Table 2. Various approximations to vacancy relaxation volumes in units of $\Omega$ according to the equations indicated. Nearest-neighbour displacements and relaxation energies used in these equations are taken from Matthai and Bacon (1985). The Eshelby factor $\gamma_{E}$ is included for convenience.

\begin{tabular}{|c|c|c|c|c|c|c|c|c|}
\hline \multirow[b]{2}{*}{ Metal } & \multirow{2}{*}{$\begin{array}{l}\text { Equation } \\
\text { (23) }\end{array}$} & \multirow[b]{2}{*}{$V_{\text {selt }}^{\text {rol }}$} & \multirow[b]{2}{*}{$\gamma_{B}$} & \multicolumn{4}{|c|}{ Equation } & \multirow[b]{2}{*}{$V_{\mathrm{rel}}^{(1) \dagger}$} \\
\hline & & & & (25) & (26) & (27) & (29) $\ddagger$ & \\
\hline $\begin{array}{c}\mathrm{V} \\
\mathrm{Nb} \\
\mathrm{Ta} \\
\mathrm{Cr} \\
\mathrm{Mo} \\
\mathrm{W}\end{array}$ & $\begin{array}{l}-0.49 \\
-0.61 \\
-0.40 \\
-0.01 \\
-0.20 \\
-0.14\end{array}$ & $\begin{array}{l}-0.25 \\
-0.28 \\
-0.21 \\
-0.03 \\
-0.13 \\
-0.11\end{array}$ & $\begin{array}{l}1.405 \\
1.290 \\
1.466 \\
1.776 \\
1.630 \\
1.687\end{array}$ & $\begin{array}{l}-0.58 \\
-0.54 \\
-0.72 \\
-0.32 \\
-0.21 \\
-0.34\end{array}$ & $\begin{array}{l}-0.73 \\
-1.16 \\
-0.48 \\
-0.44 \\
-0.29 \\
-0.33\end{array}$ & $\begin{array}{l}-0.23 \\
-0.20 \\
-0.31 \\
-0.18 \\
-0.11 \\
-0.17\end{array}$ & $\begin{array}{l}-0.32 \\
-0.31 \\
-0.34 \\
-0.18 \\
-0.11 \\
-0.16\end{array}$ & $\begin{array}{l}-0.31 \\
-0.34 \\
-0.28 \\
-0.05 \\
-0.14 \\
-0.13\end{array}$ \\
\hline
\end{tabular}

† From table 1 .

$\ddagger$ With $c=1$.

and subsequent addition to a surface kink position) and observe that the volume derivative of the second term is compensated by the external Cauchy pressure, which holds the perfect crystal at the correct lattice spacing. Thus,

$$
\frac{V_{\mathrm{rel}}^{(0)}}{\Omega}=-\frac{2 P_{\mathrm{c}}}{B}
$$

as derived by Miller and Heald (1975) from lattice statics. The remarkable feature of eqn. (23) is that a point-defect property is a function of macroscopic elastic properties only. By contrast, for the FS-potentials eqn. (19) may be transformed into

$$
\frac{V_{\mathrm{rel}}^{(0)}}{\Omega}=-\frac{1}{B}\left(\frac{-u_{N} P_{\mathrm{c}}}{2 \Omega}\right)^{1 / 2}
$$

with a different $P_{\mathrm{c}}$ dependence and an additional dependence on a microscopic property, namely the $N$-body part of the cohesive energy per atomic volume. Comparison of eqns. (23) and (24) shows poor agreement (see first and second column of table 2), although we notice the common trend of increasing $V_{\mathrm{rel}}^{(0)}$ with increasing Cauchy pressure. Since at present non-equilibrium potentials are generally regarded as unsatisfactory (see, for example, FS), eqn. (24) should certainly be preferred to eqn. (23).

Other attempts to estimate vacancy relaxation volumes treat displacements of nearest neighbours by means of elasticity theory. Most simply, the volume increase of the sphere with the nearest neighbours on its surface is taken to be the volume change $\Delta V^{\infty}$ of an infinite crystal. The additional volume change of the corresponding finite crystal is included via the Eshelby factor $\gamma_{E}$ (see Maysenhölder 1984), leading to

$$
V_{\mathrm{rel}}^{(1)}=\gamma_{E} \Delta V^{\infty} \text {. }
$$

Alternatively, using the continuum Green tensor function $\mathbf{G}$, one may write

$$
V_{\mathrm{rel}}^{(1)}=-\frac{s(r) \cdot(\nabla \cdot G(r))}{B(\nabla \cdot G(r))^{2}}
$$

where $s(r)$ denotes the displacement of an atom at position $\mathbf{r}$ (cf. Leibfried and Breuer 1978 , p. 145). $\mathbf{G}$ and $\nabla \cdot \mathbf{G}$ are known analytically for the main symmetry directions in cubic crystals (Leibfried and Breuer 1978, N. Breuer 1981, personal communication). 
Columns 4 and 5 of table 2 show the results from eqns. (25) and (26), respectively, evaluated with the relative displacements of nearest neighbours given by Matthai and Bacon (1985). For comparison $V_{\mathrm{rel}}^{(1)}$ from table 1 is listed again in the last column of table 2. Obviously, the results from both eqns. (25) and (26), which would be exact for an elastic continuum, exceed the value $V_{\text {rel }}^{(1)}$ from table 1 significantly, in the case of chromium even by factors of 6 and 9 . This is clearly due to the appreciable deviations of the atomic displacements from their continuum approximations in the immediate vicinity of the vacancy. This inadequacy is illustrated even more drastically by the nextnearest neighbour displacements as given by Matthai and Bacon (1985), which point away from the vacancy and would lead to positive relaxation volumes. $(r \cdot \nabla \cdot G$ can be positive for the $\langle 100\rangle$-directions, e.g. for copper, but is negative for all b.c.c. transition metals.)

Alternatively, we can derive a lowest-order approximation from another method described in Schober and Ingle (1980) which determines Kanzaki forces from the displacements in region I via the force constant matrix of the perfect crystal. For $N=1$ it is easily shown that

$$
V_{\mathrm{rel}}^{(1)}=\frac{\delta E_{\delta \delta}^{p}}{3 B}
$$

with

$$
E_{\delta \delta}^{p}=E_{\delta \delta}+\left\{\begin{array}{l}
8 \tilde{W}_{1} \\
12 \tilde{W}_{1} \\
8 \tilde{X}_{1}+2 A f^{-1}\left(7 \Gamma_{1}^{2}+3 \Gamma_{1} \Gamma_{2}\right)
\end{array}\right.
$$

for pair potentials in b.c.c. and f.c.c. lattices and Finnis-Sinclair potentials in b.c.c. lattices, respectively. As in the method of $\S 2$, higher-order approximations converge quickly to the exact result. However, the resuits of eqn. (12) are clearly better than those of eqn. (27): for the examples of $\S 3$ with $\delta$ according to eqn. (10) we obtain $-0.11 \Omega$ (modified Morse) and $-0.30 \Omega$ (Johnson 1964), which is far too large in both cases. The results for the Finnis-Sinclair potentials (see table 2) have been evaluated with the $\delta s$ from Matthai and Bacon (1985).

Finally we discuss the possibility of estimating vacancy relaxation volumes from the relaxation energy $E_{\text {rel }}$, which is assumed to equal the negative of the elastic energy $E_{\mathrm{el}}$ of a continuum with a spherical hole of volume $c \Omega$. Following Kröner (1954) we get

$$
E_{\mathrm{el}}=\frac{\gamma_{E}-1}{2 \gamma_{E}^{2}} \frac{P_{0}^{2}}{B C \Omega}
$$

with $P_{0}=\frac{1}{3} \operatorname{tr} P$ and arrive at

$$
\frac{V_{\mathrm{rel}}}{\Omega} \approx-\left(\frac{2 c \gamma_{E}^{2} E_{\mathrm{rel}}}{\left(\gamma_{E}-1\right) B \Omega}\right)^{1 / 2}
$$

It is not clear how large the hole should be for optimal simulation of a vacancy. Reasonable volumes may range from one atomic volume up to the volume of the 'nearest-neighbour sphere' $(\approx 5.44 \Omega$ for a b.c.c. lattice). With $c=1$ we obtain (except for chromium) surprising similarity with $V_{\text {rel }}^{(1)}$ from table 1 (see table 2), but this must be considered accidental. Although the results from eqn. (29) with $c=1$ may be not unreasonable in many cases (the numbers are $-0.11,-0.21$ and -0.34 for $\mathrm{Cu}, \mathrm{Ag}$ and 
$\mathrm{Au}$, respectively), they can be very misleading as in the case of chromium (cf. also the discussion of related problems in the palladium-hydrogen system by Bass, Oates, Schober and Stoneham 1984).

In conclusion we propose to use eqns. (9) and (12) for zeroth and first-order approximations to vacancy relaxation volumes. These estimates are theoretically well founded, do not require information on the relaxed configuration and can be calculated relatively quickly.

\section{ACKNOWLEDGMENT}

The author is grateful to Professor A. Seeger for a critical reading of the manuscript.

\section{REFERENCES}

Bass, R., OAtes, W. A., Schober, H. R., and Stoneram, A. M., 1984, J. Phys. F, 14, 2869.

Bauer, R., MAYSENhÖLDer, W., and SeEger, A., 1982, Phys. Lett., 90 A, 55 and unpublished results.

Dederichs, P. H., LehmanN, C., Schober, H. R., Scholz, A., and Zeller, R., 1978, J. nucl. Mater., $69+70,176$

EHRHART, P., 1978, J. nucl. Mater., 69+70, 200.

FINNIS, M. W., and SACHDEv, M., 1976, J. Phys. F, 6, 965.

Finnis, M. W., and Sinclair, J. E., 1984, Phil. Mag. A, 50, 45.

Foreman, J. E., 1963, Phil. Mag., 8, 1211.

HATCHER, R. D., ZelleR, R., and DeDERIChS, P. H., 1979, Phys. Rev. B, 19, 5083.

Johnson, R. A., 1964, Phys. Rev., 134, A1329.

Kanzaki, H. J., 1957, J. Phys. Chem. Sol., 2, 24.

KRŐNER, E., 1954, Acta metall., 2, 302.

Leibfried, G., and Breuer, N., 1978, Point Defects in Metals I (Berlin: Springer).

Matthai, C. C., and Bacon, D. J., 1985, Phil. Mag. A, 52, 1.

MAYSENHÖlder, W., 1984, Phys. Lett., 100 A, 289.

MAYSENhŌlder, W., BAuUR, R., and SeEger, A., 1985, Phys. Lett., 109 A, 393.

Miller, K. M., and Heald, P. T., 1975, Phys. Stat. Sol. (b), 67, 569.

SChober, H. R., and Ingle, K. W., 1980, J. Phys. F, 10, 575.

SCHOBER, H. R., and ZelLER, R., 1978, J. nucl. Mater., 69+70, 341. 